\title{
OPEN Sleep disordered breathing and subjective excessive daytime sleepiness in relation to the risk of motor vehicle crash: the Toon Health Study
}

\author{
Ryotaro Matsuo ${ }^{1}$, Takeshi Tanigawa ${ }^{2 \bowtie}$, Kiyohide Tomooka ${ }^{1}$, Ai Ikeda $^{1}$, Hiroo Wada ${ }^{2}$, \\ Koutatsu Maruyama ${ }^{3}$ \& Isao Saito ${ }^{4}$
}

Sleep disordered breathing (SDB) is a significant cause of motor vehicle crash (MVC). We conducted a prospective cohort study among 1047 Japanese community-dwellers to detect whether the presence or absence of subjective excessive daytime sleepiness (EDS) affect the association of SDB with a risk of MVC. SDB was assessed by a single airflow monitor which measured the respiratory disturbance index (RDI) during one-night, and participants were classified into the SDB group (RDI $\geq 10$ ) and nonSDB group (RDI < 10). Subjective EDS was defined as Japanese version of Epworth Sleepiness Scale scores $\geq 11$. A follow-up questionnaire five years after the baseline ascertained history of MVC over the period. Multivariable logistic regression analysis examined the association between SDB and MVC after stratification by subjective EDS. The multivariable-adjusted odds ratios ( $95 \%$ confidence interval) for MVC among the female SDB group were 1.66 (1.05-2.63) compared with the non-SDB group, and this association was more evident in females without subjective EDS [1.84(1.02-3.32)], but not among those with subjective EDS. There was no significant association in males. These findings indicate that SDB screening should be recommended regardless of subjective EDS to prevent SDB-related MVC among general population, particularly in females.

Sleep disordered breathing (SDB) is characterized by episodes of apneas and hypopneas during sleep and this condition is widely prevalent in the general population ${ }^{1}$. The prevalence of SDB (apnea-hypopnea index $(\mathrm{AHI}) \geq 15)$ was $13 \%$ of men and $6 \%$ of women in the U.S. ${ }^{2}$, and $27.1 \%$ of men and $11.1 \%$ of women in $\operatorname{Japan}^{3}$. Clinical evidence suggests that SDB is a risk factor for motor vehicle crashes (MVCs) ${ }^{4-6}$, and a meta-analysis concluded that patients with SDB have a crash risk approximately 2.5 times that of individuals without SDB ${ }^{5}$. Moreover, the treatment with continuous positive airway pressure reduces the risk of $\mathrm{MVCs}^{7}$. Thus, early detection and prevention of SDB carries safety significance ${ }^{8}$.

Also, subjective excessive daytime sleepiness (EDS) is widely known as a risk factor for $\mathrm{MVCs}^{9}$, and is one of the diagnostic criteria of sleep apnea syndrome ${ }^{10}$. However, a previous study reported no significant difference in AHI score between the SDB patients with and without EDS ${ }^{11}$. Other study also reported that there are relatively many SDB patients who do not have subjective EDS in general population ${ }^{12}$; the reason for this discrepancy may be due to subjective assessment of EDS being underestimated by chronic sleep deprivation ${ }^{13}$ and differences among individuals ${ }^{11}$.

While a large number of studies have assessed the relationship between SDB and MVC, only a prospective cohort study examined the association of sleep apnea with or without subjective EDS on the risk of MVC ${ }^{14}$. The aim of this study was to examine whether individuals with SDB were at increased risk of MVCs, independent of subjective EDS in a prospective cohort study among Japanese community dwelling population.

\footnotetext{
${ }^{1}$ Department of Public Health, Faculty of Medicine, Juntendo University, Tokyo 113-8421, Japan. ${ }^{2}$ Department of Public Health, Juntendo University Graduate School of Medicine, Tokyo 113-8421, Japan. ${ }^{3}$ Laboratory of Community Health and Nutrition, Special Course of Food and Health Science, Department of Bioscience, Graduate School of Agriculture, Ehime University, Matsuyama 790-0905, Japan. ${ }^{4}$ Department of Public Health and Epidemiology, Faculty of Medicine, Oita University, Oita 879-5593, Japan. ${ }^{\circledR}$ email: tataniga@juntendo.ac.jp
} 


\begin{tabular}{|c|c|c|c|c|c|c|}
\hline & \multicolumn{3}{|c|}{ Males } & \multicolumn{3}{|c|}{ Females } \\
\hline & \multicolumn{2}{|c|}{$\begin{array}{l}\text { RDI, } \\
\text { events/h }\end{array}$} & \multirow[b]{2}{*}{$P$ value } & \multicolumn{2}{|c|}{$\begin{array}{l}\text { RDI, } \\
\text { events/h }\end{array}$} & \multirow[b]{2}{*}{$P$ value } \\
\hline & $<10$ & $\geq 10$ & & $<10$ & $\geq 10$ & \\
\hline $\mathrm{N}$ & 123 & 257 & - & 391 & 276 & - \\
\hline Age (years) & 57.3 & 60.7 & $<0.01$ & 52.2 & 57.3 & $<0.01$ \\
\hline BMI $\left(\mathrm{kg} / \mathrm{m}^{2}\right)$ & 23.3 & 23.9 & 0.02 & 22.1 & 23.4 & $<0.01$ \\
\hline JESS (score) & 8.7 & 8.0 & 0.16 & 8.6 & 8.75 & 0.64 \\
\hline Sleeping duration $(\mathrm{h})$ & 6.6 & 6.8 & 0.18 & 6.5 & 6.4 & 0.69 \\
\hline Use of sleeping medications (\%) & 8.1 & 4.7 & 0.18 & 8.7 & 9.8 & 0.63 \\
\hline Current drinker $(\%)$ & 76.4 & 77.4 & 0.83 & 48.1 & 42.0 & 0.12 \\
\hline Current smoker (\%) & 19.5 & 14.8 & 0.24 & 4.3 & 2.5 & 0.22 \\
\hline Diabetes mellitus (\%) & 10.6 & 13.2 & 0.46 & 5.9 & 7.6 & 0.38 \\
\hline $\operatorname{MVC}(\%)$ & 16.3 & 14.4 & 0.64 & 11.8 & 16.7 & 0.07 \\
\hline
\end{tabular}

Table 1. Sex-specific characteristics of the study participants according to the category of respiratory disturbance index (RDI). Mann-Whitney U test for continuous variables. Chi-squared test for categorical variables. BMI body mass index, JESS Japanese version of Epworth Sleepiness Scale, $M V C$ motor vehicle crash.

\begin{tabular}{|l|l|l|l|l|}
\hline \multirow{2}{*}{} & \multicolumn{2}{|l|}{ Males } & \multicolumn{2}{l|}{ Females } \\
\cline { 2 - 5 } & \multicolumn{2}{l}{ JESS, score } & \multicolumn{2}{l|}{ JESS, score } \\
\cline { 2 - 5 } & $<\mathbf{1 1}$ & $\mathbf{1 1}$ & $<\mathbf{1 1}$ & $\geq \mathbf{1 1}$ \\
\hline Participants, n & 279 & 101 & 458 & 209 \\
\hline Cases, n & 37 & 20 & 56 & 36 \\
\hline Model 1 & 1.00 & $1.62(0.88-2.94)$ & 1.00 & $1.49(0.95-2.40)$ \\
\hline Model 2 & 1.00 & $1.47(0.80-2.72)$ & 1.00 & $1.50(0.94-2.40)$ \\
\hline Model 3 & 1.00 & $1.46(0.79-2.70)$ & 1.00 & $1.43(0.91-2.27)$ \\
\hline
\end{tabular}

Table 2. Odds ratios (95\% confidence intervals) of MVC according to JESS category. Model 1: adjusted for age. Model 2: adjusted for age and sleep duration. Model 3: adjusted for age and diabetes mellitus. JESS Japanese version of Epworth Sleepiness Scale, $M V C$ motor vehicle crash.

\section{Results}

The sex-specific characteristics of participants according to respiratory disturbance index (RDI) categories are shown in Table 1. Individuals with SDB, both males and females, were significantly older than those of the nonSDB group. The incidence of MVC tended to be higher among females with SDB, albeit insignificantly $(P<0.07)$.

Table 2 shows the results of multivariable-adjusted odds ratio (OR) (95\% confidence interval (CI)) for MVC according to Japanese version of Epworth Sleepiness Scale (JESS) categories. The multivariable-adjusted ORs (95\% CIs) of MVC among participants of the EDS group were $1.47(0.80-2.72)$ for males, and $1.50(0.94-2.40)$ for females, compared with non-EDS groups.

Table 3 shows the results of multivariable-adjusted OR (95\% CI) for MVC according to RDI categories. The multivariable-adjusted ORs (95\% CIs) of MVC among participants of the SDB group were $0.93(0.51-1.70)$ for males, and $1.66(1.05-2.63)$ for females, compared with non-SDB groups $(P$ for interaction $=0.38)$. This association was also examined after stratification by the presence or absence of EDS (Table 4). The multivariableadjusted ORs (95\% CIs) of MVC among participants of the SDB group were 1.84 (1.02-3.32) for females free of EDS, whereas 1.44 (0.69-3.00) for females with EDS, compared with the respective participants of the non-SDB groups. The results also showed a borderline significant effect for EDS on the association between SDB and MVC $(P$ for interaction $=0.07)$. No such association was found in males.

\section{Discussion}

The major finding of the present study is to demonstrate the significant association between SDB and risk of MVC in female participants, and this association was more evident among those without subjective EDS. A previous prospective cohort study of 3201 community residents in the United States showed an increase in OR for MVC by $15 \%$ for every 10 -unit increase in $\mathrm{AHI}$ in the overall population and by $17 \%$ for every 10 -unit increase in $\mathrm{AHI}$ in participants who did not report subjective $\mathrm{EDS}^{14}$. In this study, the significant association was found only among female in the whole analysis, but not among males. However, we could not find any significant effect modification of sex on the association between SDB and MVC. The possible reason of this sex difference was that the number of male participants in this study was relatively small to detect the association between SDB and MVC.

In this study, $76 \%$ of male and $70 \%$ of female participants with SDB did not have subjective EDS. A previous large community-based study; the Sleep Heart Health Study also reported that $54.3 \%$ of their SDB participants 


\begin{tabular}{|l|l|l|l|l|}
\hline & \multicolumn{2}{|l|}{ Males } & \multicolumn{2}{l|}{ Females } \\
\cline { 2 - 5 } & \multicolumn{2}{|l|}{ RDI, events/h } & \multicolumn{2}{l|}{ RDI, events/h } \\
\cline { 2 - 5 } & $<\mathbf{1 0}$ & $\mathbf{1 0}$ & $<\mathbf{1 0}$ & $\geq \mathbf{1 0}$ \\
\hline Participants, n & 123 & 257 & 391 & 276 \\
\hline Cases, $\mathrm{n}$ & 20 & 37 & 46 & 46 \\
\hline Model 1 & 1.00 & $0.92(0.50-1.67)$ & 1.00 & $1.66(1.05-2.62)$ \\
\hline Model 2 & 1.00 & $0.93(0.51-1.70)$ & 1.00 & $1.66(1.05-2.63)$ \\
\hline Model 3 & 1.00 & $0.92(0.50-1.68)$ & 1.00 & $1.65(1.05-2.61)$ \\
\hline
\end{tabular}

Table 3. Odds ratios (95\% confidence intervals) of MVC according to RDI category. Model 1: adjusted for age. Model 2: adjusted for age, sleep duration. Model 3: adjusted for age and diabetes mellitus. MVC motor vehicle crash, $R D I$ respiratory disturbance index.

\begin{tabular}{|c|c|c|c|c|}
\hline & \multicolumn{2}{|c|}{ Males } & \multicolumn{2}{|c|}{ Females } \\
\hline & \multicolumn{2}{|c|}{ RDI, events/h } & \multicolumn{2}{|c|}{ RDI, events/h } \\
\hline & $<10$ & $\geq 10$ & $<10$ & $\geq 10$ \\
\hline \multicolumn{5}{|l|}{ JESS $<11$} \\
\hline Participants, $\mathrm{n}$ & 85 & 194 & 265 & 193 \\
\hline Cases, $\mathrm{n}$ & 11 & 26 & 27 & 29 \\
\hline Model 1 & 1.00 & $1.11(0.51-2.40)$ & 1.00 & $1.82(1.01-3.27)$ \\
\hline Model 2 & 1.00 & $1.14(0.52-2.47)$ & 1.00 & $1.84(1.02-3.32)$ \\
\hline Model 3 & 1.00 & $1.11(0.51-2.40)$ & 1.00 & $1.82(1.01-3.28)$ \\
\hline \multicolumn{5}{|l|}{$\mathrm{JESS} \geq 11$} \\
\hline Participants, $\mathrm{n}$ & 38 & 63 & 126 & 83 \\
\hline Cases, $\mathrm{n}$ & 9 & 11 & 19 & 17 \\
\hline Model 1 & 1.00 & $0.68(0.25-1.84)$ & 1.00 & $1.45(0.70-3.03)$ \\
\hline Model 2 & 1.00 & $0.68(0.25-1.84)$ & 1.00 & $1.44(0.69-3.00)$ \\
\hline Model 3 & 1.00 & $0.66(0.24-1.79)$ & 1.00 & $1.47(0.70-3.08)$ \\
\hline
\end{tabular}

Table 4. Odds ratio (95\% confidence intervals) of MVC according to RDI stratified by presence of excessive daytime sleepiness. Model 1: adjusted for age. Model 2: adjusted for age and sleep duration. Model 3: adjusted for age and diabetes mellitus. RDI respiratory disturbance index, JESS Japanese version of Epworth Sleepiness Scale, $M V C$ motor vehicle crash.

did not have subjective EDS respectively even among those with moderate to severe SDB $(\mathrm{AHI} \geq 15)^{15}$. These results suggest that there is a relatively higher proportion of individual with SDB who do not have EDS among general population.

Previous studies discussed the potential of subjective EDS as the cause of MVC 9 . In this study, subjective EDS was borderline significantly associated with increased risk of MVC in female participants. The causes of EDS are multifactorial, e.g., shortage of nocturnal sleep length, frequent sleep fragmentation ${ }^{16}$, dysfunction of circadian rhythm ${ }^{17}$, psychiatric disorders, diabetes ${ }^{18}$. Therefore, in this study, the independent association between SDB and MVC among females with EDS might be attenuated by these other factors which might causes EDS.

Furthermore, previous studies showed that the decline in vigilance was significantly associated with $\mathrm{SDB}^{19}$, and affected fatigue-related accidents, including $\mathrm{MVCs}^{20}$. On the other hand, other studies showed that discrepancy between awareness of subjective sleepiness and vigilance due to the consequences of chronic sleep deficiency has been reported ${ }^{13}$; suggesting that subjective EDS is less perceived to be due to cumulative sleep deficiency. In addition, a previous study reported that increased cortical activation during the task of auditory psychomotor vigilance significantly predicted poor driving performance among SDB patients ${ }^{21}$. Thus, a decline in vigilance, which affects driving performance, is significantly associated with SDB, rather than with subjective EDS. Our findings suggest that SDB is a risk factor for MVC, irrespective of subjective EDS. Therefore, screening for SDB even among individuals without subjective EDS might be significant for prevention of MVC.

The present study has several strength points. These include participants relatively high follow-up rate (69\%) over 5 years, and it was conducted as a prospective cohort study among community residents and demonstrated the association of SDB with MVC after adjustment for potential confounding factors. Apart from these strengths, the study also has several limitations. First is that, the experience of MVC was based on retrospective self-administered data, therefore it potentially contains reporting bias. However, the Toon Health Study is administered in voluntary community residents. Compared with questionnaires administered by the employers as part of the medical examination (in Japan, employees undergo annual health checkups by the employers), there are no potential disadvantages or repercussions (e.g., losing jobs, increase in insurance premiums) by reporting MVC. Therefore, we believe that the participants declared their MVC experience correctly in the self-reported 
questionnaire in this study. Second is that one-channel flow-sensor device was used to assess the severity of SDB instead of polysomnography (PSG). However, the reliability and validity of the device was fairly good and quite comparable with full PSG tests ${ }^{22,23}$. Thirdly, although there might be other potential confounding factors on the association between EDS and MVC, e.g., dysfunction of circadian rhythm ${ }^{17}$, psychiatric disorders ${ }^{18}$, these variables were not available in this study. Further studies are needed in order to consider these issues.

In conclusion, we have demonstrated in the present study a significant association between SDB and MVC among females, and this association was more evident among those without EDS than those with EDS. SDB screening is strongly recommended regardless of EDS to prevent SDB-related MVC among community dwelling population.

\section{Methods}

Study subject. This study was conducted as a part of the Toon Health Study which is a community-based prospective cohort study to characterize the risk factors for cardiovascular disease in Toon City, Ehime prefecture, Japan ${ }^{24,25}$. In the present study, we recruited participants from approximately 22,000 residents of Toon city aged $\geq 30$ to $<80$ years using newspaper advertisements, posters, and direct invitations. At the baseline survey from 2009 to 2012, 2032 participants were enrolled in this study and 1395 participants were followed up approximately 5 years later. Of these, participants with incomplete data on overnight airflow monitor $(n=153)$ were excluded. We also excluded non-drivers $(n=166)$, those with a history of stroke $(n=27)$ and participants with missing data on sleep duration $(n=2)$. Thus, the data of 1047 (males $=380$, females $=667$ ) individuals were eligible for analysis.

The study protocol was approved by The Institutional Review Board of Ehime University Graduate School of Medicine and the Ethics Committee of Juntendo University, and written informed consent was obtained from each study participant. All methods in this study were performed in accordance with the relevant guidelines and regulations.

Motor vehicle crash history. In a self-administered questionnaire completed approximately 5 years after the baseline, the participants were asked "Do you usually drive?" Those who responded affirmatively were also asked questions on MVC; "Have you ever had an accident while driving related to sleepiness in the past five years?" and "Have you ever had an accident while driving regardless of sleepiness in the past five years?" Participants who answered affirmatively for either of these two questions were defined as those who have had MVC during the five-year period.

Assessment of sleep disordered breathing. The participants in this study completed a general sleep questionnaire and screening of SDB. To determine the presence or absence of SDB, a single-channel portable monitor; "Somnie" (NGK Spark Plug Co., Nagoya, Japan) ${ }^{22,23}$ was used during one night of sleep at home. The device records nasal/oral airflow with a polyvinylidene fluoride film sensor taped below the nose and has the capacity to store $24 \mathrm{~h}$ recording of the airflow signal. The morning after the recording, the participant completed a self-administered questionnaire about sleep state the night before. The data stored in the "Somnie" were subsequently analyzed by the "Flow" software (Institute of Sleep Health Promotion, Tokyo, Japan), which uses short-time power spectral analysis to compute the RDI. In this study, the criterion for SDB was defined by RDI level of 10 events per hour, and participants were classified as either SDB group or non-SDB. We have previously validated the "Somnie" device and confirmed that this RDI cutoff value represents AHI of $\geq 15$ events per hour as determined by full PSG; with a corresponding sensitivity and specificity of 0.91 and $0.82^{22}$. We showed the prevalence of SDB among 5320 Japanese males by using the single-channel portable monitor ${ }^{26}$.

Assessment of covariates. Height and weight were measured in stocking feet with light clothing. BMI was calculated as weight $(\mathrm{kg})$ divided by the square of height $\left(\mathrm{m}^{2}\right)$. Trained dietitian asked the participants to assess their drinking (never, former, or current drinker) and smoking (never, former or current smoker) habits. To assess EDS, each participant completed the JESS questionnaire, which is the sum of the scores of eight items (each from 0 to 3, total score range, $0-24$ ). A JESS score of $\geq 11$ represented the presence of EDS ${ }^{27}$. Sleep duration and use of sleeping medications were assessed using part of the Pittsburg Sleep Quality Index ${ }^{28}$. We also measured serum glucose levels during fasting and after the oral glucose tolerance test. All participants were required to fast for at least $10 \mathrm{~h}$ before the oral glucose tolerance test. The reference starch solution (Toleran-G75, Ajinomoto Pharma Co., Ltd., Tokyo, Japan.) containing $75 \mathrm{~g}$ of glucose was orally administered in a fasting state. Venous blood samples were obtained at baseline, 1 and $2 \mathrm{~h}$ after the ingestion of $75 \mathrm{~g}$ glucose. Diabetes mellitus was defined as either a fasting serum glucose level of $\geq 7.0 \mathrm{mmol} / \mathrm{L}$, a $2 \mathrm{~h}$ post-load glucose level of $\geq 11.1 \mathrm{mmol} / \mathrm{L}$ or those receiving anti-diabetic medication.

Analysis. In this study, we conducted sex-stratified analyses, because the proportion of RDI $\geq 10$ events per hour was different between the two sexes (males $=69.2 \%$, females $=41.6 \%$ ). To analyze the differences between participants, the Mann-Whitney $U$ test was used for continuous variables and chi-square test was used for dichotomous variables according to the RDI categories. Multivariable logistic regression analysis were used to calculate the ORs and 95\% CIs for the MVC during the 5 years period according to JESS and RDI categories at baseline. Furthermore, stratified analysis was performed by the presence of EDS. The interaction of SDB with the presence of EDS in relation to MVC was tested using cross-product terms of these variables in the logistic regression model. Multivariable adjusted models were conducted using following confounding factors; age (year) in model 1, age (year) and sleep duration (h) in model 2, age (year) and diabetes mellitus (yes/no) in model 3. All 
statistical analyses were performed using SAS version 9.4 (SAS Institute, Inc., Cary, NC). All $P$ values were twotailed, and values $<0.05$ were considered statistically significant.

Received: 15 May 2020; Accepted: 21 September 2020

Published online: 12 October 2020

\section{References}

1. Malhotra, A. \& White, D. P. Obstructive sleep apnoea. Lancet 360, 237-245 (2002).

2. Peppard, P. E. et al. Increased prevalence of sleep-disordered breathing in adults. Am. J. Epidemiol. 177, 1006-1014 (2013).

3. Yamagishi, K. et al. Cross-cultural comparison of the sleep-disordered breathing prevalence among Americans and Japanese. Eur. Respir. J. 36, 379-384 (2010).

4. Ellen, R. L. et al. Systematic review of motor vehicle crash risk in persons with sleep apnea. J. Clin. Sleep Med. 2, 193-200 (2006).

5. Tregear, S., Reston, J., Schoelles, K. \& Phillips, B. Obstructive sleep apnea and risk of motor vehicle crash: Systematic review and meta-analysis. J. Clin. Sleep Med. 5, 573-581 (2009).

6. Tregear, S., Reston, J., Schoelles, K. \& Phillips, B. Continuous positive airway pressure reduces risk of motor vehicle crash among drivers with obstructive sleep apnea: Systematic review and meta-analysis. Sleep 33, 1373-1380 (2010).

7. Burks, S. V. et al. Nonadherence with employer-mandated sleep apnea treatment and increased risk of serious truck crashes. Sleep 39, 967-975 (2016).

8. Filomeno, R., Ikeda, A. \& Tanigawa, T. Significance of early detection and treatment of sleep apnea syndrome. Juntendo Med. J. 63, 422-426 (2017).

9. Quera-Salva, M. A. et al. Association between reported sleep need and sleepiness at the wheel: Comparative study on French highways between 1996 and 2011. BMJ Open 6, e012382 (2016).

10. American Academy of Sleep Medicine. International Classification of Sleep Disorders 3rd edn. (American Academy of Sleep Medicine, Darien, 2014).

11. Ryan, C. M., Kendzerska, T., Wilton, K. \& Lyons, O. D. The different clinical faces of obstructive sleep apnea (OSA), OSA in older adults as a distinctly different physiological phenotype, and the impact of OSA on cardiovascular events after coronary artery bypass surgery. Am. J. Respir. Crit. Care Med. 192, 1127-1129 (2015).

12. Garbarino, S. et al. Obstructive sleep apnea with or without excessive daytime sleepiness: Clinical and experimental data-driven phenotyping. Front. Neurol. 9, 505 (2018).

13. Van Dongen, H. P., Maislin, G., Mullington, J. M. \& Dinges, D. F. The cumulative cost of additional wakefulness: Dose-response effects on neurobehavioral functions and sleep physiology from chronic sleep restriction and total sleep deprivation. Sleep 26, $117-126(2003)$.

14. Gottlieb, D. J., Ellenbogen, J. M., Bianchi, M. T. \& Czeisler, C. A. Sleep deficiency and motor vehicle crash risk in the general population: A prospective cohort study. BMC Med. 16, 44 (2018).

15. Kapur, V. K. et al. Sleepiness in patients with moderate to severe sleep-disordered breathing. Sleep 28, 472-477 (2005).

16. Gillberg, M. et al. Sleepiness and its relation to the length, content, and continuity of sleep. J. Sleep Res. 4, 37-40 (1995)

17. Pavlova, M. Circadian rhythm sleep-wake disorders. CONTINUUM: Lifelong Learn. Neurol. 23, 1051-1063 (2017).

18. Bixler, E. O. et al. Excessive daytime sleepiness in a general population sample: The role of sleep apnea, age, obesity, diabetes, and depression. J. Clin. Endocrinol. Metab. 90, 4510-4515 (2005).

19. Tanno, S. et al. Sleep-related intermittent hypoxia is associated with decreased psychomotor vigilance in Japanese community residents. Sleep Med. 29, 7-12 (2017).

20. Abe, T., Mollicone, D., Basner, M. \& Dinges, D. F. Sleepiness and safety: Where biology needs technology. Sleep Biol. Rhythms 12, 74-84 (2014).

21. Vakulin, A. et al. Individual variability and predictors of driving simulator impairment in patients with obstructive sleep apnea. J. Clin. Sleep Med. 10, 647-655 (2014).

22. Nakano, H. et al. Validation of a single-channel airflow monitor for screening of sleep-disordered breathing. Eur. Respir. J. 32, 1060-1067 (2008).

23. Nakano, H., Tanigawa, T., Furukawa, T. \& Nishima, S. Automatic detection of sleep-disordered breathing from a single-channel airflow record. Eur. Respir. J. 29, 728-736 (2007).

24. Tanno, S. et al. Sleep-related intermittent hypoxemia and glucose intolerance: A community-based study. Sleep Med. 15, 1212-1218 (2014).

25. Saito, I. et al. Heart rate variability, insulin resistance, and insulin sensitivity in Japanese adults: The Toon Health Study. J. Epidemiol. 25, 583-591 (2015).

26. Cui, R. et al. Associations between weight change since 20 years of age and sleep-disordered breathing among male truck drivers. Int. J. Obes. (Lond.) 33, 1396-1401 (2009).

27. Takegami, M. et al. Development of a Japanese version of the Epworth Sleepiness Scale (JESS) based on item response theory. Sleep Med. 10, 556-565 (2009).

28. Mollayeva, T. et al. The Pittsburgh sleep quality index as a screening tool for sleep dysfunction in clinical and non-clinical samples: A systematic review and meta-analysis. Sleep Med. Rev. 25, 52-73 (2016).

\section{Acknowledgements}

We thank the staff and participants of the Toon Health Study and the municipal authorities, officers, and health professionals of Toon City for their valuable contributions. This study was supported in part by Grants-in-Aid for Scientific Research (Grants-in-Aid for Research B, \#22390134 for 2010-2012, \#25293142 for 2013-2015 and \#18H03056 for 2018-2022) from the Ministry of Education, Culture, Sports, Science and Technology of Japan, and Health, and Labor Sciences Research Grants (Comprehensive Research on Life-Style Related Diseases including Cardiovascular Diseases and Diabetes Mellitus, \#201021038A for 2010-2012) from the Ministry of Health, Welfare and Labor, Japan.

\section{Author contributions}

R.M. collected and analyzed the data and drafted the manuscript. I.S., K.M. and T.T. designed and coordinated the study and obtained funding. R.M., K.M. and K.T. collected the data. A.I. and K.T. provided technical assistance for data analysis. I.S., T.T., A.I., K.M., H.W. and K.T. interpreted the data and critically revised the manuscript. T.T. is a guarantor for this work. 


\section{Competing interests}

The authors declare no competing interests.

\section{Additional information}

Correspondence and requests for materials should be addressed to T.T.

Reprints and permissions information is available at www.nature.com/reprints.

Publisher's note Springer Nature remains neutral with regard to jurisdictional claims in published maps and institutional affiliations.

(c) (1) Open Access This article is licensed under a Creative Commons Attribution 4.0 International License, which permits use, sharing, adaptation, distribution and reproduction in any medium or format, as long as you give appropriate credit to the original author(s) and the source, provide a link to the Creative Commons licence, and indicate if changes were made. The images or other third party material in this article are included in the article's Creative Commons licence, unless indicated otherwise in a credit line to the material. If material is not included in the article's Creative Commons licence and your intended use is not permitted by statutory regulation or exceeds the permitted use, you will need to obtain permission directly from the copyright holder. To view a copy of this licence, visit http://creativecommons.org/licenses/by/4.0/.

(c) The Author(s) 2020 